3. Hayward JS, Eckerson JD, Kemna D. Thermal and cardiovascular changes during three methods of resuscitation from mild hypothermia. Resuscitation. 1984;11:21-33.

4. Giesbrecht GG, Bristow GK. The convective afterdrop component during hypothermic exercise decreases with delayed exercise onset. Aviat Space Environ Med. 1998;69:17-22.

5. Guyatt G, Gutterman D, Baumann MH, et al. Grading strength of recommendations and quality of evidence in clinical guidelines: report from an American College of Chest Physicians Task Force. Chest. 2006;129:174-181.

6. Althaus U, Aeberhard P, Schupbach P, Nachbur BH, Muhlemann W. Management of profound accidental hypothermia with cardiorespiratory arrest. Ann Surg. 1982;195:492-495.

7. Stoneham MD, Squires SJ. Prolonged resuscitation in acute deep hypothermia. Anaesthesia. 1992;47:784-788.

8. Giesbrecht GG, Hayward JS. Problems and complications with cold-water rescue. Wilderness Environ Med. 2006;17:26-30.

9. Giesbrecht GG, Bristow GK. A second postcooling afterdrop: more evidence for a convective mechanism. J Appl Physiol. 1992;73:1253-1258.

\section{The Hand-Powered Ring Cutter: A Useful Tool in Your Wilderness Medical Bag}

\section{To the Editor:}

A gentleman in his mid-40s presented with a left fourth finger injury to the medical team at the finish line of a 50-mile mountain bike race. He reported dislocation of his left fourth proximal interphalangeal joint (PIPJ) as a result of a fall during the race. He was able to self-reduce his finger immediately. Despite severe pain and swelling, he completed the race. On arrival to the medical station, there was no joint deformity, but he had significant edema of his fourth finger on examination, with a ring that was not removable. He was very tender over the PIPJ, and the neurovascular condition of the finger was intact. The ring was removed using a hand-powered ring cutter (Figure).

Finger injuries are among the most common musculoskeletal injuries in endurance athletes. If the injury happens while the athlete is wearing a ring, immediate removal of the ring is important to prevent constriction syndrome. ${ }^{1}$ Most cases of digital constriction (tourniquet) syndromes occur in infants and mentally ill patients. ${ }^{2,3}$ Delay in presenting for medical attention may happen for a variety of reasons. ${ }^{2-4}$ Etiologies of digital tourniquet syndrome include external constricting bands (eg, metal, plastic, hair) and traumatic, infectious, endocrinologic, allergic, rheumatologic, and dermatologic conditions. ${ }^{1,2}$ Treatment of an acute edematous finger with a constricting ring requires urgent intervention. Neurovascular examination of the affected digit is important. The ring can be removed with less-invasive methods like distal

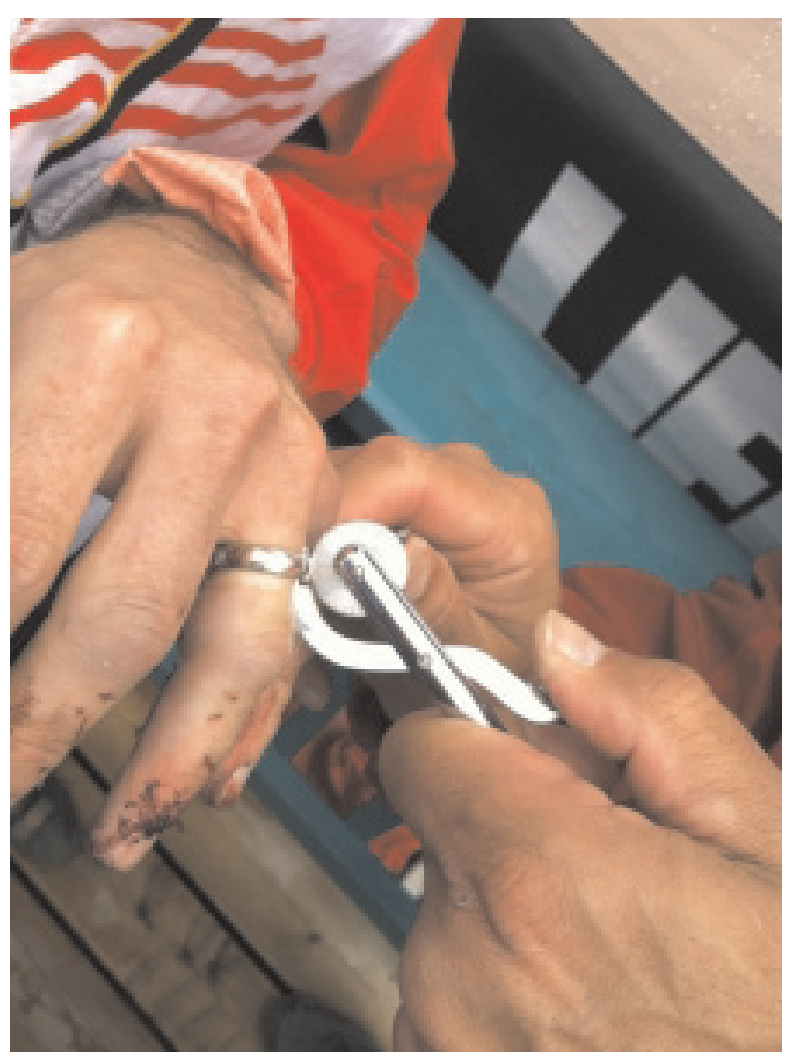

Figure. Removal of the trapped ring by a hand-powered ring cutter.

lubrication (eg, soap, mineral oil, and lubricant gel), distal edema reduction (eg, elevation of the affected extremity, ice, compression of the distal area to the ring), sequential compression (eg, dental floss, sutures, elastic tapes, and string-wrap methods), and surgical glove method. ${ }^{1,5}$ If these methods are unavailable or unsuccessful, division of the constricting band is necessary. ${ }^{1,5}$ This can be achieved by a cutting tool or a saw (hand-powered or motorized). A hand-powered ring cutter is an easy-to-use tool that can be added to a medical bag as in our case.

\section{Acknowledgment}

The authors would like to thank Mr. George Gipson.

Morteza Khodaee, MD, MPH Jill Tirabassi, MD

University of Colorado School of Medicine Denver, CO, USA

\section{References}

1. Peckler B, Hsu CK. Tourniquet syndrome: a review of constricting band removal. J Emerg Med. 2001;20:253-262. 
2. Kumar A, Edwards H, Lidder S, Mestha P. Dangers of neglect: partially embedded ring upon a finger. BMJ Case Rep. 2013 May 9;2013.

3. Shankar R, Rao PS, Shetty KB, Sahu P, Singal R, Gupta S. Digital strangulation in a child: tourniquet syndrome. Indian J Dermatol Venereol Leprol. 2012;78:408.

4. Kang N, Gault D. The real ring constriction syndrome. $\mathrm{Br} J$ Plast Surg. 2000;53:634.

5. Haynes JH, Haynes AT, Hines TS. Ring removal from an edematous finger. In: Pfenninger JL, Fowler GC, eds. Pfenninger and Fowler's Procedures for Primary Care. Philadelphia, PA: Elsevier; 2011:215-217.

\section{Acute Stress Symptoms Among US Ocean Lifeguards}

\section{To the Editor:}

We designed a survey to study ocean lifeguard responses after a lifesaving rescue. Respondents were generally "healthy," physically active Caucasian men, most being from California. During their career, $48 \%$ of respondents reported acute stress symptoms after performing such a rescue, especially those with a history of anxiety or a history of asthma.

During the past century, ocean lifeguards have grown to play a critical role in protecting swimmers at many US beaches. They perform lifesaving ocean rescues and medical interventions. Approximately a quarter of American adults visit the beach annually.

The health of lifeguards is potentially at risk as a result of long hours of daily seawater exposure through skin contact, water ingestion, or inhalation of marine aerosolized particles, and the psychological stress of rescues. A limited number of studies have considered sun exposure, red tides, drowning, rescues, fitness standards, visual acuity, and swimming performance. However, no studies, to our knowledge, have examined acute stress symptoms because of occupational rescues by ocean lifeguards.

The goal of our study was to obtain data from lifeguards about their health and acute stress symptoms experienced associated with ocean rescues as a lifeguard. The objective of this study was to determine whether ocean rescues were associated with acute stress symptoms.

English-speaking US ocean lifeguards at least 18 years old and employed a minimum of 20 hours per week were eligible to participate in the survey. Recruitment occurred through the United States Lifeguard Association online message board, social media ads, and by fliers. Study participants provided informed consent online and completed an online questionnaire using the software Survey Monkey (Survey Monkey, Inc, Palo Alto, CA).
The survey was conducted from August through November 2010.

Data analysis was conducted using Stata 11 statistical software package (StataCorp LP, College Station, TX). Univariate analysis and multivariate logistic regression were used to quantitatively describe the associations between risk factors and acute stress symptoms. The final multivariate logistic model included the independent variables of physician diagnosis of anxiety and asthma and self-reported adverse health symptoms after exposure to a red tide. The risk of illness was described as an adjusted risk ratio, with the associated $95 \%$ confidence interval (CI). The Institutional Review Board of the University of California, Berkeley approved the study protocol (201005-1557).

One hundred sixty-eight ocean lifeguards responded to our survey. Table 1 reports lifeguard characteristics.

Nearly half the lifeguards reported at least 1 acute stress symptom after an ocean rescue (Table 2). Lifeguards experienced acute stress symptoms, but did not meet diagnostic criteria for acute stress disorder. In the adjusted multivariate logistic regression, lifeguards with a history of anxiety were 1.7 times more likely to experience acute stress symptoms after a rescue than lifeguards without a history of anxiety (95\% CI, 1.3 to 2.2). Lifeguards who reported adverse health symptoms after red tide exposure were 1.4 times more likely to experience acute stress after a rescue than lifeguards without such a history (95\% CI, 1.1 to 1.9$)$. Lifeguards with a history of asthma were 1.7 times more likely to experience acute stress symptoms after a rescue as compared with lifeguards without a history of asthma (95\% CI, 1.3 to 2.1 ).

This is the first study to examine acute stress symptoms among ocean lifeguards. This was a relatively young, healthy, and active cohort. Nearly half reported acute stress symptoms after an ocean rescue. Our study found an association between lifeguard acute stress symptoms after an ocean rescue and anxiety, asthma, and adverse health symptoms after red tide exposure.

Anxiety is one of the most prevalent mental health problems in the United States, estimated at $18 \%$ of the US adult population. " "Acute stress disorder" is classified as an anxiety disorder in the Diagnostic and Statistical Manual of Mental Disorders. ${ }^{2}$ Given the potential life-threatening nature of lifeguard duties, ocean rescues for some lifeguards can be an occupational stressor leading to acute stress symptoms.

Stress has been identified as a biological trigger for asthma in the general US population. ${ }^{3}$ Interventions to decrease asthma symptoms need to address individual reactions to acute stress. The self-report of adverse health 